\title{
Expression and Prognostic Role of E2F2 in Hepatocellular Carcinoma
}

\author{
Shen Shen $\mathbb{1 D}^{1,2}$ \\ Yanfang Wang ${ }^{3}$
}

'Gene Hospital of Henan Province, Precision Medicine Center, The First Affiliated Hospital of Zhengzhou University, Zhengzhou, Henan, 450052, People's Republic of China; ${ }^{2}$ Department of Infectious Diseases, The First Affiliated Hospital of Zhengzhou University, Zhengzhou, Henan Province, People's Republic of China; ${ }^{3}$ Department of Pharmacy, The First Affiliated Hospital of Zhengzhou University, Zhengzhou, 450052, People's Republic of China

Correspondence: Shen Shen

Gene Hospital of Henan Province, Precision Medicine Center, The First Affiliated Hospital of Zhengzhou University, No. I Jianshe Road,

Zhengzhou, 450052, Henan, People's

Republic of China

Email fccshenk@zzu.edu.cn
Introduction: Hepatocellular carcinoma (HCC) is a common clinical malignancy. Recent studies reported that E2F transcription factor 2 (E2F2) plays a significant role in tumor progression. However, its expression and biological function in HCC are still unclear. Therefore, we explored the relationship between E2F2 expression and tumor progression in HCC.

Methods: In this study, we utilized some online tools to explore the E2F2 expression in pancarcinoma and HCC. The association of E2F2 expression with the clinical characteristics and prognosis of HCC was further studied. In addition, we explored the co-expressed genes of E2F2 and mined the positively/negatively corrected significant genes and excavated the possible functions. Meanwhile, the hub gene set was constructed based on protein-protein interaction (PPI) network, and the relationship between E2F2 and immunity was discovered. Results: We observed that the expression level of E2F2 was generally upregulated in HCC. However, E2F2 expression was not significantly different between HCC and normal tissues in regard to the disease stage 4 . Furthermore, we also observed the poor prognosis in patients with high E2F2 expression. The co-expressed genes of E2F2 were identified and further detected. Thereafter, we identified the positively/negatively corrected significant genes and constructed the hub gene network of E2F2 based on PPI network. We also found that E2F2 expression was positively correlated with the infiltration levels of CD4+ T, CD8 $+\mathrm{T}$ cells, macrophages, neutrophils, and dendritic cells.

Conclusion: Our findings suggested that E2F2 could be a potential prognostic factor for HCC, which could provide a therapeutic target for the molecular treatment of HCC.

Keywords: E2F2, hepatocellular carcinoma, prognosis, hub genes, diagnosis

\section{Introduction}

Hepatocellular carcinoma (HCC) is one of the six most common tumors in the world, which ranks third in mortality among all malignancies. ${ }^{1}$ Despite advances in prevention, screening, diagnosis, and treatment techniques, its morbidity and mortality are still increasing. ${ }^{2}$ Surgical resection, liver transplantation, and chemotherapy are still the main therapeutic HCC approaches. ${ }^{3}$ Molecular-targeted drugs have demonstrated to extend survival for advanced $\mathrm{HCC}$, which cannot be surgically resected. ${ }^{4}$ However, existing treatment strategies are still not efficient in controlling the progression of HCC and preventing its recurrence. Therefore, the study of gene expression related to $\mathrm{HCC}$ is expected to fundamentally understand the development of liver cancer at the molecular level and carry out targeted prevention and treatment.

The pathogenesis of HCC remains unclear along with the lack of targeted therapies. Numerous studies aimed to explore its pathogenesis from the point of view of molecular biology in order to discover new therapeutic targets. Previous 
studies have illustrated that miR22HG, as a tumor suppressor gene, significantly inhibits the proliferation, invasion, and in vivo and in vitro metastasis of HCC cells. ${ }^{5}$ External miR-451a has demonstrated to play an anticancer role in HCC by targeting LPIN $1 .{ }^{6}$ Xiang also found that oncofetal HLF transactivates c-Jun to promote $\mathrm{HCC}$ development. ${ }^{7}$ More and more studies are being conducted on genes involved in HCC; however, these studies are not enough.

E2F transcription factors play an important role in the cell cycle and influence the apoptosis of cells through the cyclin-dependent kinase (CDK)-RB-E2F axis, which plays a vital role in the occurrence of tumors. ${ }^{8}$ E2F transcription factor 2 (E2F2) is a member of the E2F family, and its abnormal expression has been shown to play an important role in the pathogenesis of various tumors. In non-small cell lung cancer (NSCLC), ${ }^{9}$ E2F2 may play a key role in the pathological mechanism of NSCLC progression through Circ_0109320/miR-595/E2F2 axis. In colon cancer tissues, ${ }^{10}$ the expression level of E2F2 was significantly higher than that in normal colon tissues. Functional enrichment analysis also found a potential correlation between E2F2 and prostate cancer. ${ }^{11}$ However, the expression, prognosis, and biological function of $\mathrm{E} 2 \mathrm{~F} 2$ in $\mathrm{HCC}$ are still uncertain.

In this study, we maximized online tools to explore the relationship between the high expression of E2F2 in $\mathrm{HCC}$ and the prognosis of patients. We also analyzed the coexpression of E2F2 and investigated the related genes positively and negatively correlated with E2F2. We developed the hub gene set based on protein-protein interaction (PPI) network. Gene Ontology (GO) and Kyoto Encyclopedia of Genes and Genomes (KEGG) were used to excavate the possible functions of E2F2. The relationship between E2F2 and tumor immunity was also evaluated, providing new ideas on $\mathrm{HCC}$ treatment.

\section{Materials and Methods}

\section{Expression and Clinical Analysis of E2F2 in $\mathrm{HCC}$}

Ualcan database (http://ualcan.path.uab.edu/analysis.html) is an effective online analysis and mining site for cancer data based on The Cancer Genome Atlas (TCGA, https:// tcga-data.nci.nih.gov/tcga/). ${ }^{12}$ We made use of Ualcan to analyze the expression of E2F2 in $371 \mathrm{HCC}$ tissues and 50 adjacent tissues obtained from the TCGA database as described previously. ${ }^{13-15}$ We further explored the relationship between E2F2 expression and clinical characteristics. In the Ualcan database, tumor samples were categorized into different groups based on the clinical patient data. Disease stages were classified according to The American Joint Committee on Cancer (AJCC) cancer staging manual, ${ }^{16}$ and tumor grades were divided into four groups according to the existing grading information. ${ }^{12}$

The survival analysis for E2F2 gene was also carried out: select "survival" to enter the interface of result analysis and calculate and draw the survival curves according to different E2F2 expression groups.

\section{Gene Differential Expression Related to E2F2}

The LinkedOmics database (http://linkedomics.org/) contains data on 32 cancer types from TCGA and is a tool for TCGA database. ${ }^{17}$ It was used to obtain the significant genes positively and negatively correlated with E2F2 expression. The top 20 the positively/negatively corrected significant genes were selected to present the data in the form of clustering heat maps.

\section{GO and KEGG Analysis}

DAVID (https://david.ncifcrf.gov/) is a biological information database, which integrates the biological data and analysis tools to provide comprehensive biological function annotation information. ${ }^{18}$ We used the DAVID website for GO and KEGG analysis to describe the molecular functions (MF), biological processes (BP), and cell components (CC) in biology. Consequently, metabolic pathways were annotated to explore possible metabolic signaling pathways.

\section{PPI Network Construction and Screening of Hub Gene Sets}

The cytohHubba and MCODE plug-ins were downloaded. The input method was "Multiple proteins" in the STRING database (https://string-db.org/). The PPI network was obtained after a list of different genes was input. The most significant module was found with MCODE and displayed in Cytoscape. CytohHubba plug-in was employed to sequence genes according to degree value. Thereafter, we screened top eight hub genes and constructed a hub gene set.

\section{Co-Expression of E2F2}

The data sources and analysis options of cBioPortal database (https://www.cbioportal.org/) were from multiple 
sites, including large tumor research projects such as TCGA. ${ }^{19}$ It assembled data from 126 cancer genome studies, including data from 28,000 specimens. We used the cBioPortal database to explore the co-expression information of E2F2 gene in HCC. The significant correlation value was screened, and the gene co-expression network was constructed.

\section{Relationship Between E2F2 and Immune Infiltration}

Tumor Immune Estimation database (TIMER, https://cis trome.shinyapps.io/timer/) is a website tool set up by a Harvard University professor, which uses an RNA-Seq expression spectrum data in the identification of tumor tissue infiltration of immune cells. ${ }^{20}$ We explored the relationship between E2F2 and immunity using TIMER. The infiltration correlation between E2F2 and six immune cells (B cells, CD4 $+\mathrm{T}$ cells, CD8 $+\mathrm{T}$ cells, neutrophils, macrophages, and dendritic cells) and tumor purity were examined.

\section{Statistical Analysis}

We utilized online tools to analyze the expression and prognosis of E2F2 in HCC. $t$-test was used in Ualcan to compare the mRNA expression levels of tumors and adjacent tissues. Survival curves were plotted using Log rank test to analyze the prognosis of patients. Pearson correlation was used to determine the correlation between gene co-expression in the cBioPortal database and immune infiltration in the TIMER database. DAVID carried out gene annotation and functional enrichment online. $\mathrm{P}$ value $<0.05$ was considered statistically significant.

\section{Results}

\section{E2F2 Expression Was Elevated in HCC and Was Associated with Prognosis}

We first analyzed the expression of E2F2 between tumor and normal samples in TCGA pan-cancer based on Ualcan, and discovered that it was highly expressed in several tumors (Figure 1A), especially in HCC (Figure 1B). Subsequently, the expression levels of E2F2 were also found to be generally elevated in HCC tissues grouped by disease stage and tumor grade compared with normal tissues. E2F2 expression levels at stage 3 were the highest, and significantly higher than those in tumor tissues and normal tissues at low stage. Similarly, higher levels of E2F2 were found in advanced HCC and higher levels in grade 3 than in grade 2 and grade
1. However, the expression of E2F2 was not significantly different between stage 4 and normal tissues (Figure 1C and D). These findings were indicative of the relationship between E2F2 and the occurrence and development of HCC, which may serve as a potential indicator for HCC diagnosis. Subsequently, overall survival (OS) curve, progression-free survival (PFS) curve, disease free survival (DFS) curve and disease-specific survival (DSS) curve were used to describe the relationship between E2F2 and $\mathrm{HCC}$ prognosis (Figure 1E-H). It was found that the survival rate of $\mathrm{HCC}$ patients with high E2F2 expression was significantly lower than that of patients with low E2F2 expression (Log rank test, $P<0.05$ ), which suggests that E2F2 may be highly expressed in HCC tissues as a risk factor.

\section{The Positively/Negatively Corrected Significant Genes of E2F2}

We applied LinkedOmics to analyze E2F2-related genes, and the top 20 positively/negatively corrected significant genes were presented in the form of heat maps (Figure 2A and B). As shown in heat maps, the column represents a sample and the row represents a gene. Red indicates positive correlation and blue indicates negative correlation. Combined with the comparison of the relative abundance of E2F2 expression, top 20 significant related genes which have positive and negative correlation with E2F2 were shown.

\section{GO and KEGG Analysis of E2F2-Related Genes}

GO annotation was performed on E2F2-related genes by DAVID. In the enriched annotation of CC, the genes were mainly located in the cytoplasm. In terms of MF, the ATP binding demonstrated the high enrichment significance. The microtubule-based movement and chromosome segregation were the primary biological pathways in the BP (Figure 2CE). KEGG pathway analysis revealed that the most significant enrichment was in the cell cycle (Figure 2F), which suggests the significant role played by E2F2 in HCC metabolism.

\section{PPI Network and Hub Gene}

We investigated the protein interaction relationship in the STRING database and constructed the PPI network diagram. The top 100 protein interaction networks are presented in Figure 3A. According to degree value, top 8 hub genes were screened (Figure 3B), including CCNB1, CDCA8, CCNB2, KIF20A, TOP2A, BUB1, BUB1B, and CDK1 (Figure 3C). Moreover, a hub gene set was 


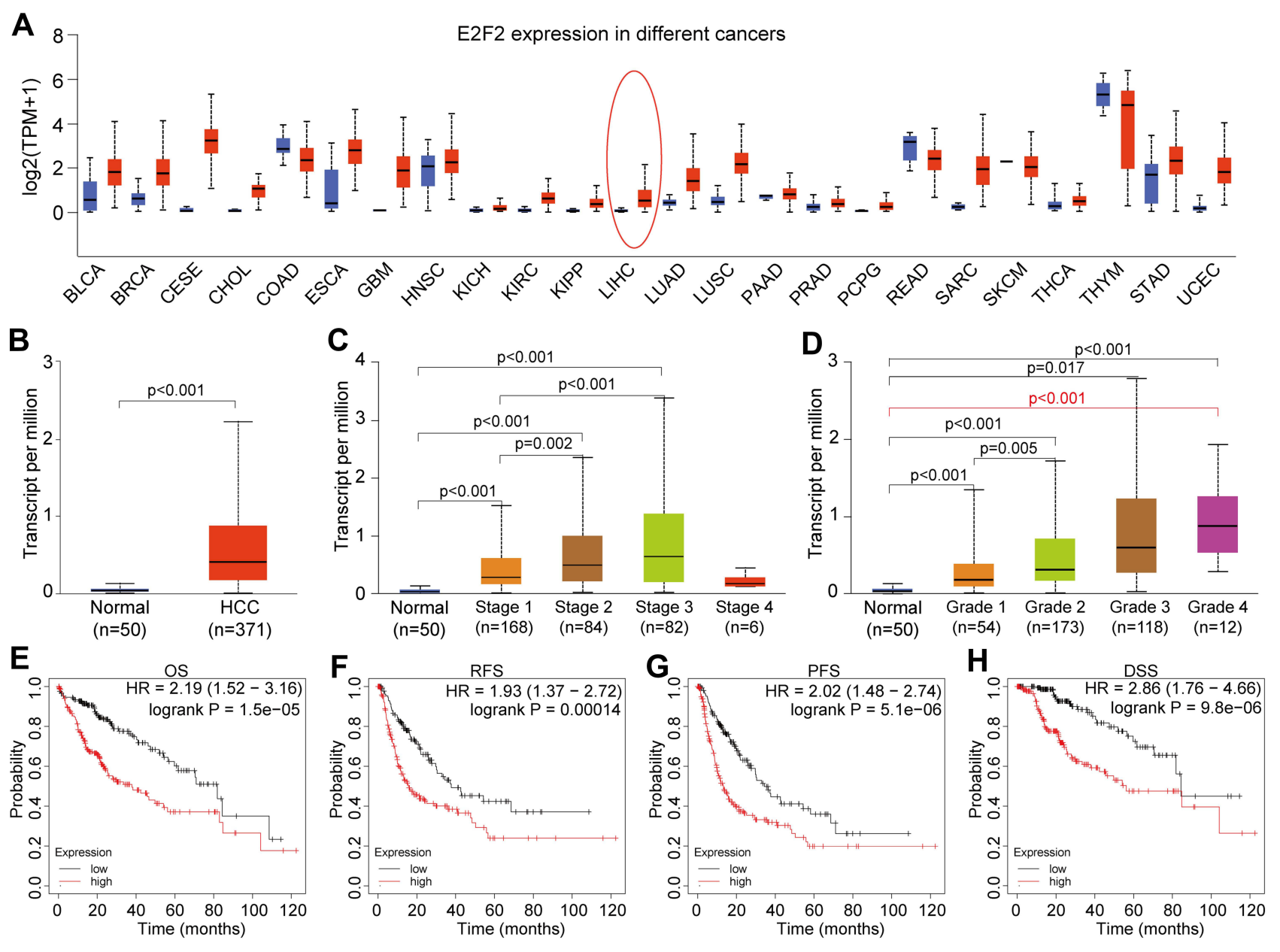

Figure I The expression of E2F2 in HCC. (A) The expression of E2F2 between tumor and normal samples in TCGA pan-cancer. (B) The expression of E2F2 in HCC tissues and adjacent tissues. (C) The difference of E2F2 expression in normal tissues and stages I, 2, 3, and 4 HCC patients. (D) The difference of E2F2 expression in normal tissues and grades I, 2, 3, and 4 HCC patients. (E-H) The OS, RFS, PPS and DSS rate of HCC patients with high E2F2 expression was significantly lower than that of patients with low E2F2 expression.

developed, which plays a crucial role in the biological process. Among these hub genes, CDCA8 ( $\mathrm{r}=0.81, P<$ $0.001)$ is probably the most significant target, while CCNB1 $(r=0.66, P<0.001)$ had the lowest correlation.

\section{The Co-Expression of E2F2}

The analysis of gene co-expression suggested that E2F2 was co-expressed with RAD54L, KIF2C, CDCA8, CDC45, RAD51, CDC20, KIF4A, ASF1B, and PKMYT1 (Figure 4A-I). Among the genes, RAD54L had the most significant relationship with E2F2. On the contrary, CDC20 had the lowest correlation.

\section{Relationship Between E2F2 and Immune Infiltration}

In TIMER, we evaluated the relationship between E2F2 and immune infiltration. Results showed that E2F2 expression was positively correlated with the six kinds of immunocytes, including $\mathrm{B}$ cells, CD4 $+\mathrm{T}$ cells, CD8+ $\mathrm{T}$ cells, macrophages, neutrophils, and dendritic cells (Figure 5A-G). The greatest correlation was with dendritic cells $(\mathrm{r}=0.489, P=7.74 \mathrm{E}-22$, Figure $5 \mathrm{G}) . \mathrm{B}$ cells $(\mathrm{r}=$ $0.463, P=1.15 \mathrm{E}-19$, Figure $5 \mathrm{~B})$ ranked second in the correlation, and CD4+ T cells $(\mathrm{r}=0.333, P=2.49 \mathrm{E}-10$, Figure 5D) ranked last.

\section{Discussion}

HCC is a typical inflammation-driven tumor, which often develops from chronic hepatitis or liver cirrhosis. $^{21}$ Regardless of factors that lead to HCC, the abnormal expression of genes and loss of control over the cell cycle will eventually induce HCC. ${ }^{22}$ In recent years, with the rapid development of high-throughput sequencing, immunotherapy and molecular targeted therapy are 
A

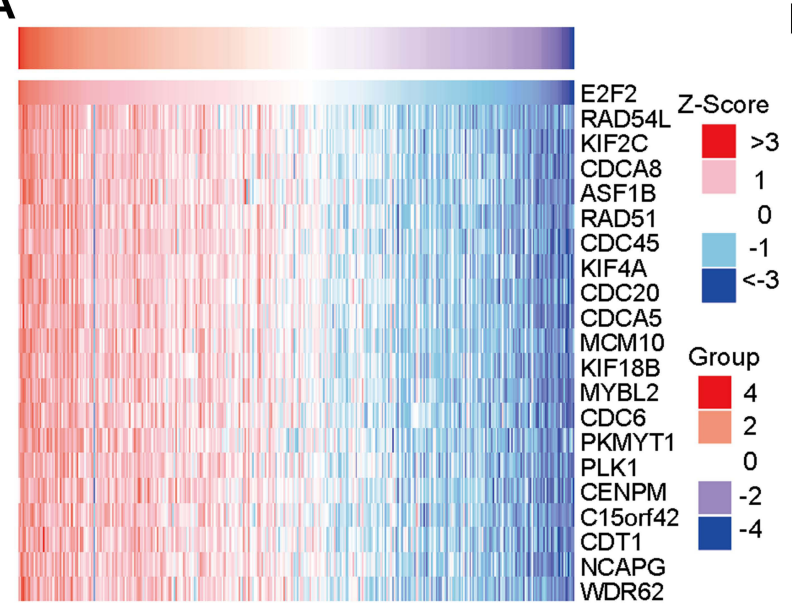

C

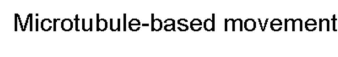

Chromosome segregation

Mitotic sister chromatid segregation

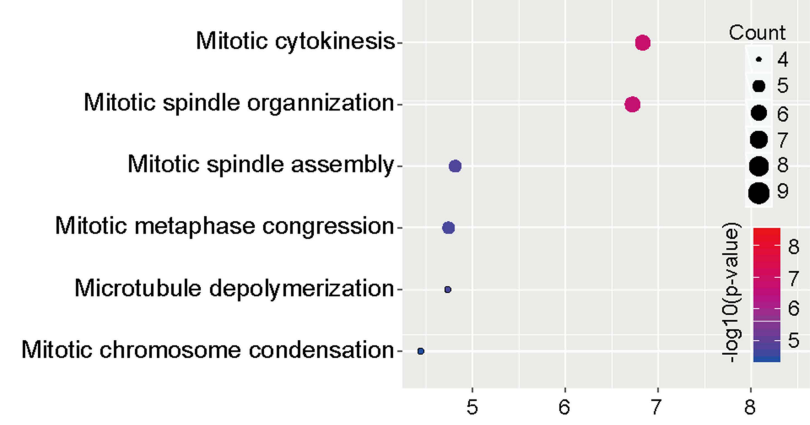

E

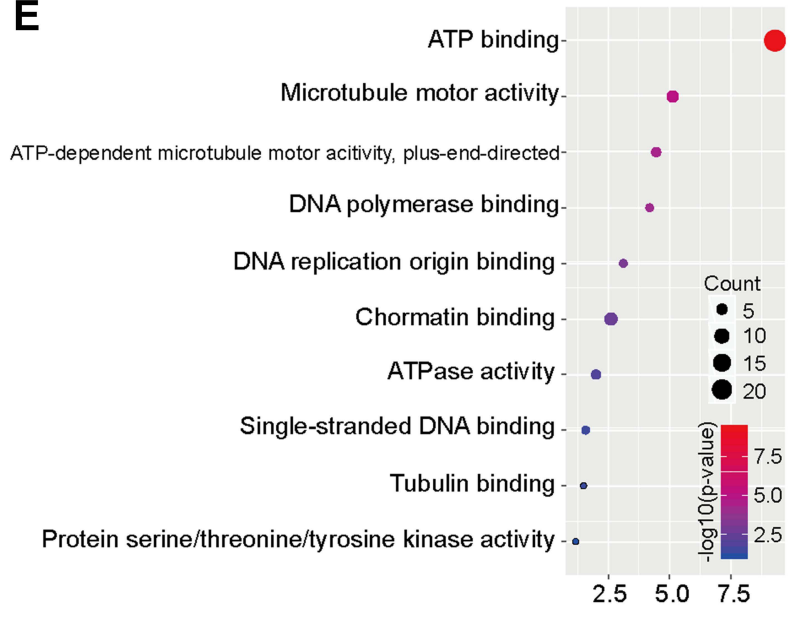

B

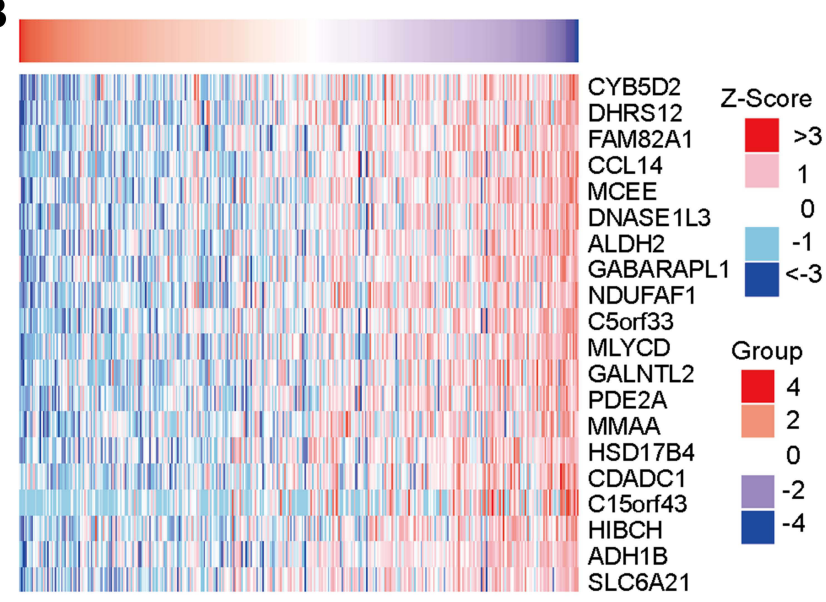

D

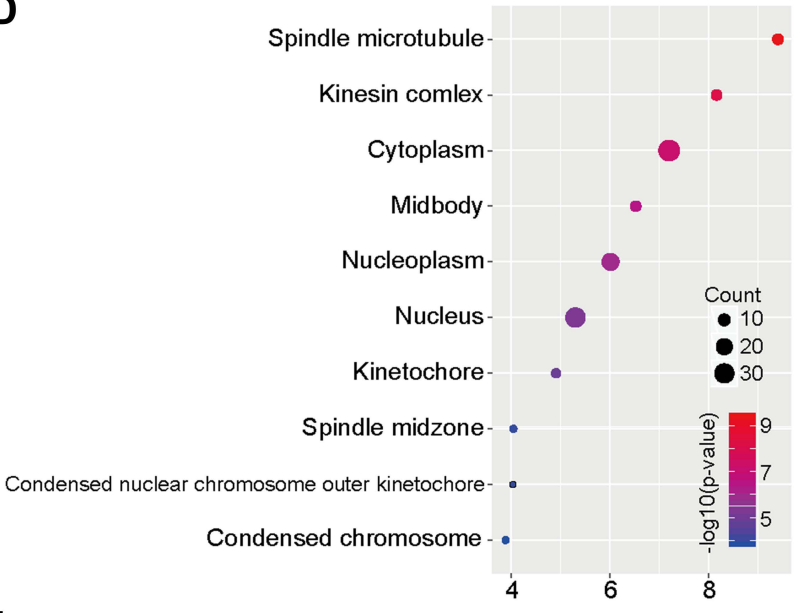

$\mathbf{F}$

Cell cycle

Rogesterone-mediated oocyte maturation

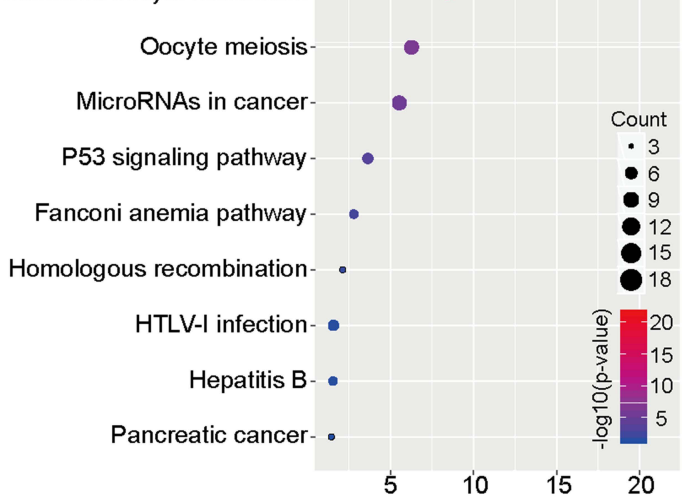

Figure 2 The gene differential expression related to E2F2. (A) The top 20 significant genes that negatively correlated with E2F2 presented in the form of heat maps. (B) The top 20 significant genes that positively correlated with E2F2. (C) GO annotation of biological process performed on E2F2-related genes by DAVID. (D) Cellular component, (E) molecular function, (F) KEGG pathway analysis.

expected to become the developmental direction of the treatment. $^{23}$

$\mathrm{E} 2 \mathrm{~F} 2$ is a member of the E2F family that can promote or inhibit the development of tumors. Upon investigating the expression of E2F2 in HCC, we discovered that the expression of E2F2 generally increased, except in HCC tissues with stage 4. E2F2 has also been reported to be overexpressed in many other human tumor cells. In high-grade 
A

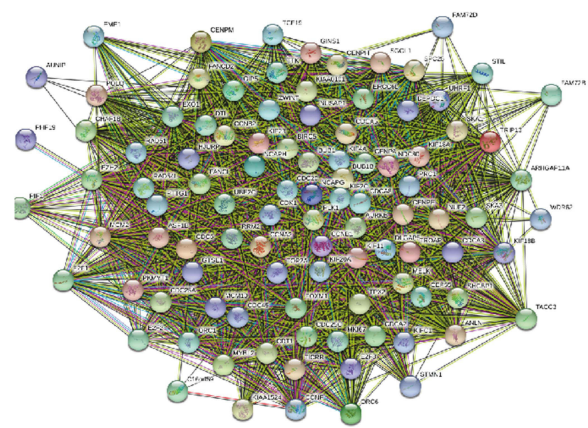

C
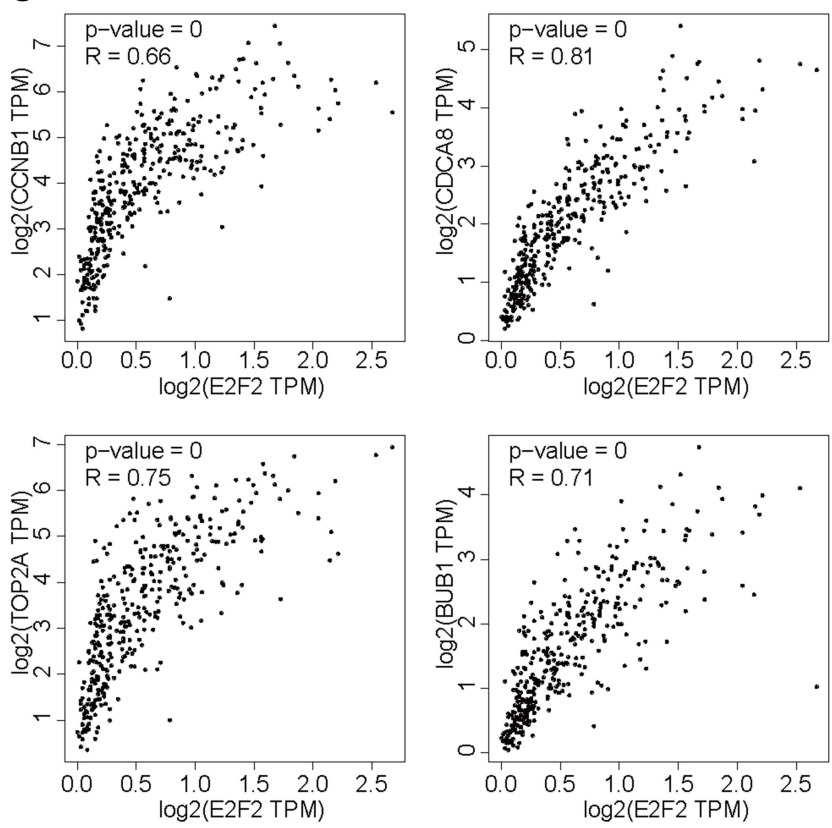
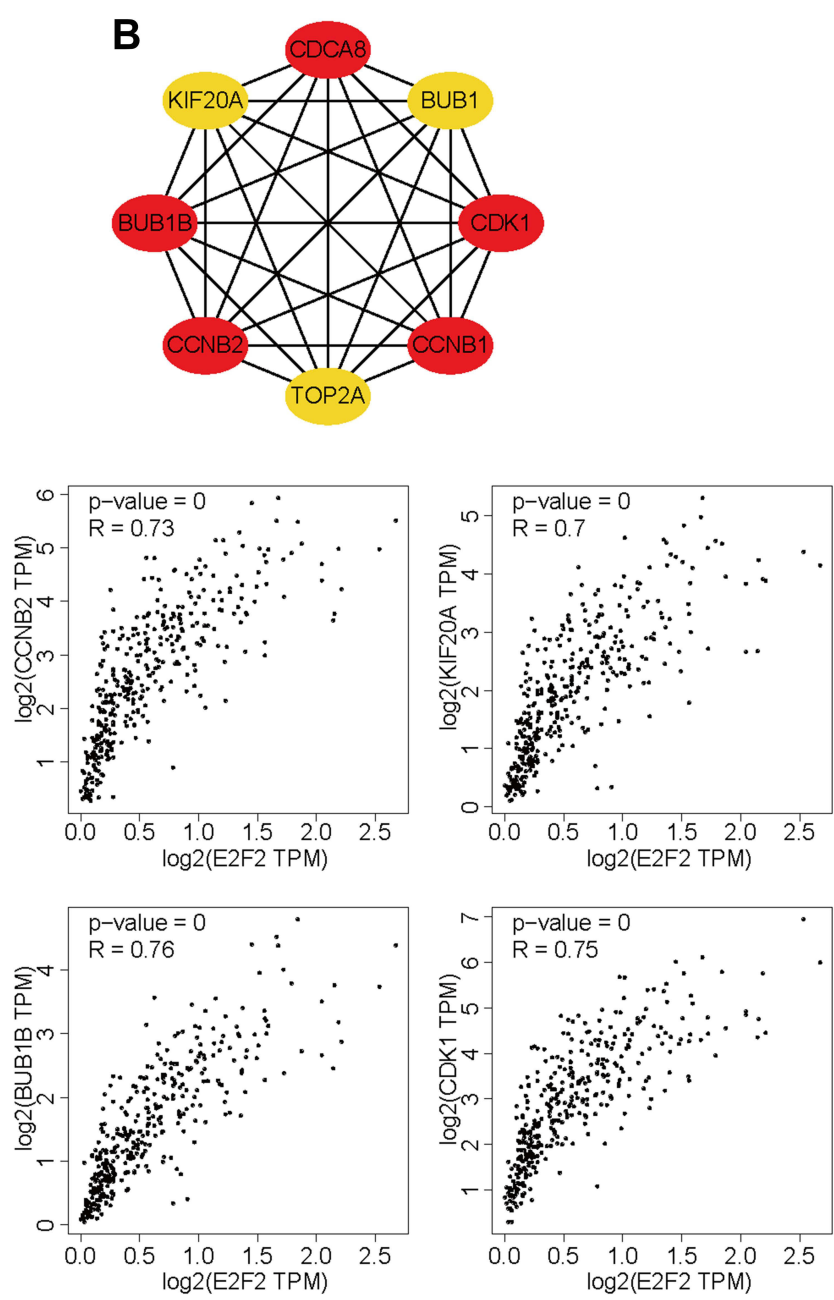

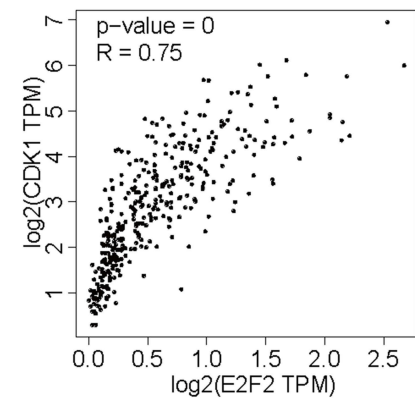

Figure 3 PPI network construction and screening of hub gene sets. (A) The protein interaction relationship was examined, and the top 100 protein interaction network was presented in the PPI network. (B) The hub gene network of E2F2 was constructed based on PPI. (C) The correlation between eight hub genes and E2F2.

glioma (HGG), ${ }^{24}$ E2F2 is highly correlated with invasive proliferative oncogenes and potential therapeutic resistance oncogenes. In bladder cancer, ${ }^{25}$ E2F2 may act as a target gene of miRNA-93 and regulate the process of apoptosis by forming DNA-binding complexes. Herein, the study suggested that the overexpression of E2F2 in $\mathrm{HCC}$ may be related to the occurrence and development of early HCC.

Eight members of the E2F transcription factor family (E2F1-8) have been identified that may be associated with a variety of cancers. ${ }^{26}$ For instance, in breast cancer, elevated E2F1, E2F3, E2F5, E2F7, and E2F8 expression levels were found to indicate poor prognosis, with decreased OS, RFS, DMF, and PPS. In addition, the high expression of E2F4 increased RFS and decreased DMFS. ${ }^{27}$ To identify the relationship between the expression of E2F2 and prognosis in $\mathrm{HCC}$, we illustrated the survival curves and found that patients with high expression have poorer prognosis than those with low expression, suggesting that high expression of E2F2 can be used for the diagnosis and prognosis of HCC.

We also constructed PPI network and screened the hub genes of HCC to further evaluate the molecular mechanism. Among the hub genes, CDCA8 $(\mathrm{r}=0.81, P<0.001)$ was most significantly correlated with E2F2. It has been proven to play a vital role in a variety of tumors and is associated with apoptosis. CDCA8 can be used as a chromosomal passenger protein to regulate cell cycle, which is related to poor prognosis of gastric cancer. ${ }^{28}$ Meanwhile, CDCA8 has been found to be related to the occurrence and development of alcohol-related HCC, with significant prognostic value. ${ }^{29}$ In bladder cancer, ${ }^{30}$ down-regulation of CDCA8 expression can inhibit the proliferation of cancer cells and enhance cell apoptosis. These studies suggested that CDCA8 may also play a synergistic role, along with E2F2 in the development and progression of 

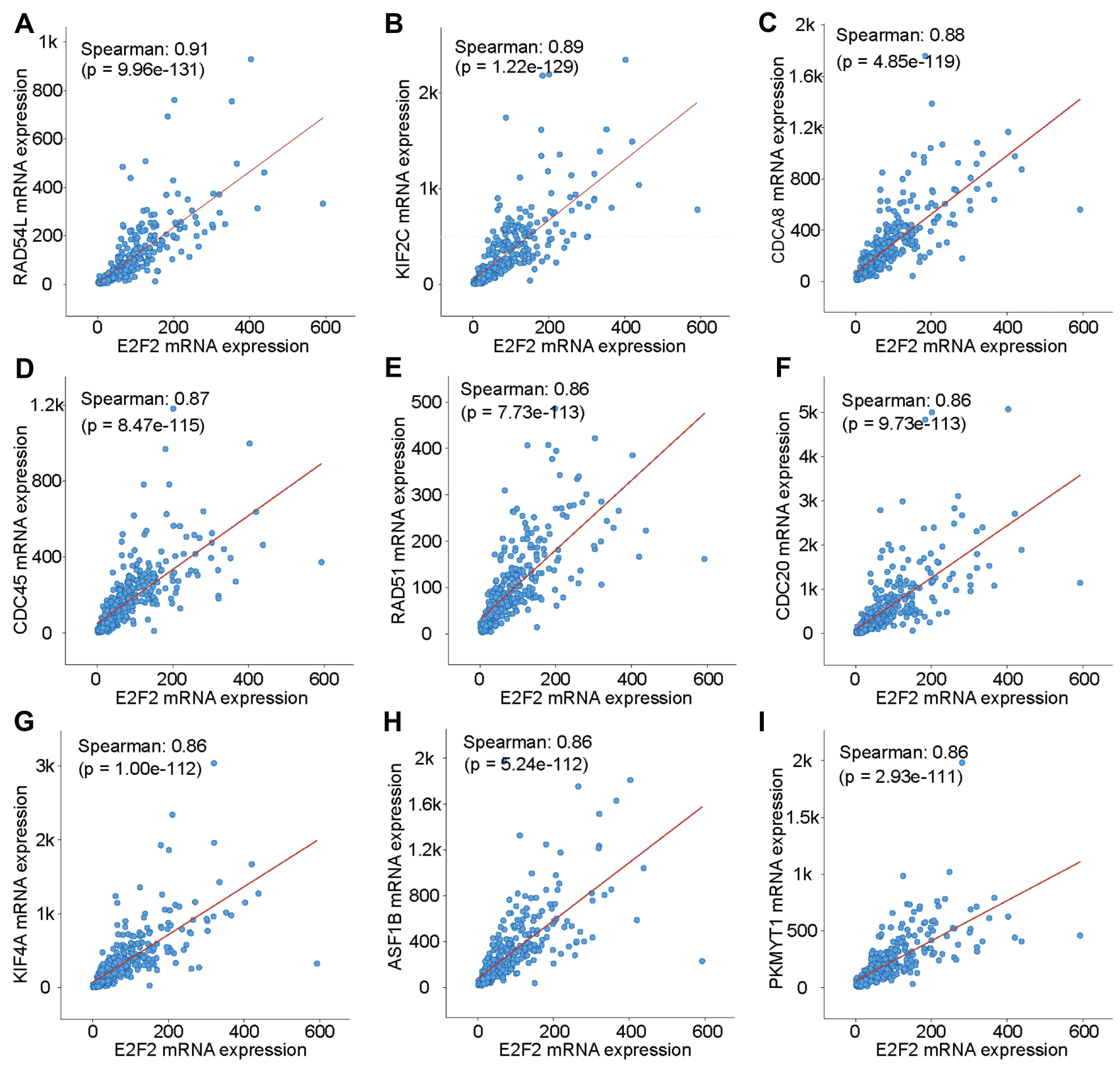

Figure 4 Co-expressed genes of E2F2. (A-I) E2F2 was co-expressed with RAD54L, KIF2C, CDCA8, CDC45, RAD5I, CDC20, KIF4A, ASFIB, and PKMYTI.

$\mathrm{HCC}$, which may be related to its role in promoting apoptosis. Moreover, BUB1B (r. $=0.76, P<0.001$ ), with the second highest correlation in our study, was believed to be associated with the poor survival of HCC patients in other study. ${ }^{31}$ Hence, research on hub genes is of great significance to the development of HCC, which is also conducive to the search for new targets for liver cancer.

With regard to the potential pathway of E2F2, the results of our study indicated that the E2F2-related genes participated in the cell circle to regulate the cell apoptosis in HCC. As is reported, the function of E2F is regulated by cyclin-dependent kinases (CDKs) and $\mathrm{RB}$, promoting cell cycle progression by participating in the (CDK)-RB-E2F axis. $^{32}$ The overexpression of E2F activator induces resting cells to enter G1 phase. A large number of studies have demonstrated that among the ten proteins encoded by eight different genes, E2F1, E2F2, and E2F3a are transcription activators. $^{33} \mathrm{G} 1 / \mathrm{S}$ transition is a key step in cell cycle control. The activator expression is increased during the G1-S phase transition. ${ }^{34}$ Changes in the activity of E2F transcription factor can regulate $\mathrm{G} 1 / \mathrm{S}$ transformation and thereby regulate cell proliferation, ${ }^{35}$ indicating that E2F2 may promote the occurrence of $\mathrm{HCC}$ by regulating cell circle. 

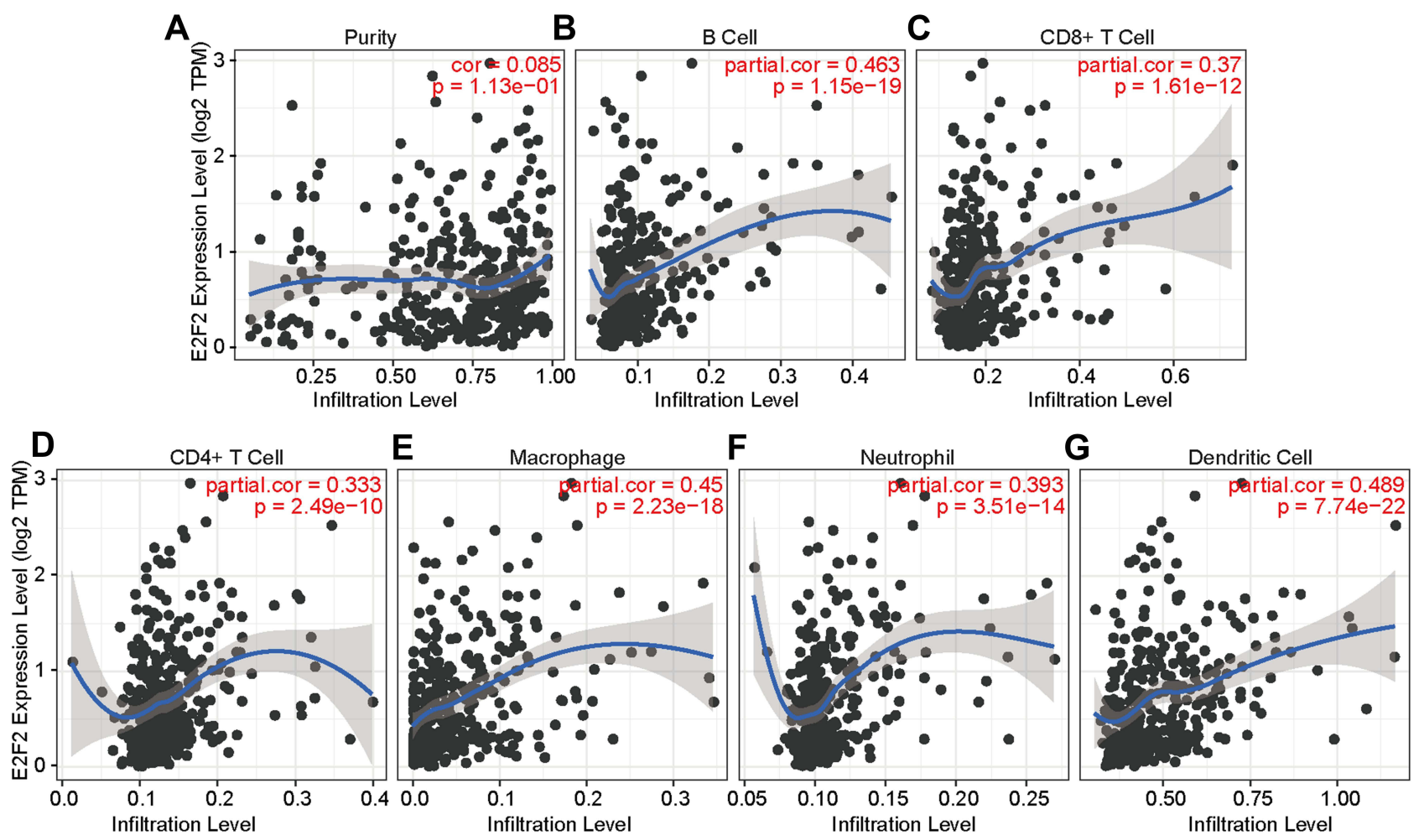

Figure 5 The relationship between E2F2 and immune infiltration. $(\mathbf{A})$ E2F2 expression has no correlation with tumor purity $(r=0.085$, $P=1.13 E-01)$. $(\mathbf{B}-\mathbf{G})$ E2F2 expression was positively correlated with the six kinds of immunocytes, including B cells, CD4+ T cells, CD8+ T cells, macrophages, neutrophils, and dendritic cells. Pearson correlation was used to determine the correlation between E2F2 and immune infiltration.

TIMER was used to explore the relationship between E2F2 and HCC immunity, and a positive correlation was found between E2F2 and CD4+ T, CD8+ T cells, B cells, macrophages, neutrophils, dendritic cells, and other infiltration levels. Since HCC induces tumor immune tolerance through various mechanisms and avoids immune killing, immunotherapy has gradually become a new developmental direction in future liver cancer treatment. ${ }^{36}$ Gao et al found that a high concentration of Tregs in HCC combined with low concentration of CD8+T cells could be an independent factor affecting the survival and recurrence of HCC patients. ${ }^{37}$ Dendritic cells have been shown to influence the prognosis of tumors in addition to their maturation and activation states, as well as their subpopulation composition. ${ }^{38}$ Moreover, macrophages also play a role in the immune microenvironment of HCC. ${ }^{39}$ Exploration of E2F2 and immune infiltration yields an idea for HCC immunotherapy.

\section{Conclusions}

Our study explored the expression and prognosis of E2F2 in HCC, investigated the potential function pathways and discovered the immunomodulatory role of E2F2. Based on current studies, we believe that E2F2 may serve as a biomarker for
HCC prognosis and is also expected to become a diagnostic and therapeutic target. In the following study, we will continue to explore the relationship between hub genes and E2F2 and reveal the regulatory relationship of E2F2. We hope to discover more molecular mechanisms and signaling pathways involved in the regulation of $\mathrm{E} 2 \mathrm{~F} 2$ on $\mathrm{HCC}$.

\section{Abbreviations}

HCC, hepatocellular carcinoma; E2F2, E2F transcription factor 2; PPI, protein-protein interaction; $\mathrm{CDK}$, cyclin-dependent kinase; NSCLC, non-small cell lung cancer; GO, Gene Ontology; KEGG, Kyoto Encyclopedia of Genes and Genomes; TCGA, The Cancer Genome Atlas; AJCC, The American Joint Committee on Cancer; MF, molecular functions; BP, biological processes; CC, cell components; TIMER, Tumor Immune Estimation; OS, overall survival; PFS, progression-free survival; DFS, disease free survival; DSS, disease-specific survival; HGG, high-grade glioma.

\section{Ethics Approval and Consent to Participate}

The study has been approved by the Ethics Committee of the First Affiliated Hospital of Zhengzhou University. 


\section{Funding}

This work was supported by the Science and Technology

Research Project of Henan Province (Grant No. 202102310115), and Henan Medical Science and Technology Joint Building Program (Grant No. LHGJ20200387).

\section{Disclosure}

The authors report no conflicts of interest in this work.

\section{References}

1. Dutta R, Mahato RI. Recent advances in hepatocellular carcinoma therapy. Pharmacol Ther. 2017;173:106-117. doi:10.1016/j. pharmthera.2017.02.010

2. Siegel RL, Miller KD, Jemal A. Cancer statistics, 2018. CA Cancer J Clin. 2018;68(1):7-30. doi:10.3322/caac. 21442

3. Galle PR, Forner A, Llovet JM, Mazzaferro V, Piscaglia F, Raoul J. EASL clinical practice guidelines: management of hepatocellular carcinoma. J Hepatol. 2018;69(1):182-236. doi:10.1016/j.jhep.2018.03.019

4. Sposito C, Mazzaferro V. The SIRveNIB and SARAH trials, radioembolization vs. sorafenib in advanced HCC patients: reasons for a failure, and perspectives for the future. Hepatobiliary Surg Nutr. 2018;7(6):487-489. doi:10.21037/hbsn.2018.10.06

5. Zhang DY, Zou X-J, Cao C-H, et al. Identification and functional characterization of long non-coding RNA MIR22HG as a tumor suppressor for hepatocellular carcinoma. Theranostics. 2018;8 (14):3751-3765. doi:10.7150/thno.22493

6. Xiang DM, Sun W, Zhou T, et al. Oncofetal HLF transactivates c-Jun to promote hepatocellular carcinoma development and sorafenib resistance. Gut. 2019;68(10):1858-1871. doi:10.1136/gutjnl-2018317440

7. Novy M, Pohn R, Andorfer P, et al. EAPP, a novel E2F binding protein that modulates E2F-dependent transcription. Mol Biol Cell. 2005;16(5):2181-2190. doi:10.1091/mbc.e04-11-0975

8. Slansky JE, Farnham PJ. Introduction to the E2F family: protein structure and gene regulation. Curr Top Microbiol Immunol. 1996;208:1-30. doi:10.1007/978-3-642-79910-5_1

9. Bai Q, Li L, Chen F, et al. Suppression of circular RNA Hsa_circ_0109320 attenuates non-small cell lung cancer progression via MiR-595/E2F7 axis. Med Sci Monit. 2020;26:e921200. doi:10.12659/MSM.921200

10. Yao H, Lu F, Shao Y. The E2F family as potential biomarkers and therapeutic targets in colon cancer. PeerJ. 2020;8:e8562. doi:10.7717/peerj. 8562

11. Wei J, Yin Y, Deng Q, et al. Integrative analysis of microRNA and gene interactions for revealing candidate signatures in prostate cancer. Front Genet. 2020;11:176. doi:10.3389/fgene.2020.00176

12. Chandrashekar DS, Bashel B, Balasubramanya SAH, et al. UALCAN: a portal for facilitating tumor subgroup gene expression and survival analyses. Neoplasia. 2017;19(8):649-658. Doi:10.1016/ j.neo.2017.05.002

13. He Y, Yu X, Li J, et al. Role of m(5) C-related regulatory genes in the diagnosis and prognosis of hepatocellular carcinoma. Am J Transl Res. 2020;12(3):912-922.

14. He Y, Dang Q, Li J, et al. Prediction of hepatocellular carcinoma prognosis based on expression of an immune-related gene set. Aging (Albany NY). 2020;12(1):965-977. doi:10.18632/aging.102669

15. Xue C, He Y, Zhu W, et al. Low expression of LACTB promotes tumor progression and predicts poor prognosis in hepatocellular carcinoma. Am J Transl Res. 2018;10(12):4152-4162.
16. Edge SB, Compton CC. The American Joint Committee on Cancer: the 7th edition of the AJCC cancer staging manual and the future of TNM. Ann Surg Oncol. 2010;17(6):1471-1474. doi:10.1245/s10434010-0985-4

17. Vasaikar SV, Straub P, Wang J, et al. LinkedOmics: analyzing multi-omics data within and across 32 cancer types. Nucleic Acids Res. 2018;46(D1):D956-D963. doi:10.1093/nar/gkx1090

18. Dennis G, Sherman BT, Hosack DA, et al. DAVID: database for annotation, visualization, and integrated discovery. Genome Biol. 2003;4(5):P3. doi:10.1186/gb-2003-4-5-p3

19. Unberath P, Knell C, Prokosch H-U, et al. Developing new analysis functions for a translational research platform: extending the cBioPortal for cancer genomics. Stud Health Technol Inform. 2019;258:46-50.

20. Li T, Fan J, Wang B, et al. TIMER: a web server for comprehensive analysis of tumor-infiltrating immune cells. Cancer Res. 2017;77(21): e108-e110. doi:10.1158/0008-5472.CAN-17-0307

21. Dimitroulis D, Damaskos C, Valsami S, et al. From diagnosis to treatment of hepatocellular carcinoma: an epidemic problem for both developed and developing world. World $J$ Gastroenterol. 2017;23(29):5282-5294. doi:10.3748/wjg.v23.i29.5282

22. Marquardt JU, Andersen JB, Thorgeirsson SS. Functional and genetic deconstruction of the cellular origin in liver cancer. Nat Rev Cancer. 2015;15(11):653-667. doi:10.1038/nrc4017

23. Sun W, Cabrera R. Systemic treatment of patients with advanced, unresectable hepatocellular carcinoma: emergence of therapies. J Gastrointest Cancer. 2018;49(2):107-115. doi:10.1007/s12029-018-0065-8

24. Yu H, Li Z, Wang M. Expression and prognostic role of E2F transcription factors in high-grade glioma. CNS Neurosci Ther. 2020;26 (7):741-753. doi:10.1111/cns.13295

25. Wang $X$, Ding Y, Wang J, et al. Identification of the key factors related to bladder cancer by IncRNA-miRNA-mRNA three-layer network. Front Genet. 2019;10:1398. doi:10.3389/fgene.2019.01398

26. Wang $\mathrm{H}$, Wang $\mathrm{X}, \mathrm{Xu} \mathrm{L}$, et al. Integrated analysis of the E2F transcription factors across cancer types. Oncol Rep. 2020;43 (4):1133-1146. doi:10.3892/or.2020.7504

27. Li Y, Huang J, Yang D, et al. Expression patterns of E2F transcription factors and their potential prognostic roles in breast cancer. Oncol Lett. 2018;15(6):9216-9230. doi:10.3892/ol.2018.8514

28. Chang JL, Chen T-H, Wang C-F, et al. Borealin/Dasra B is a cell cycle-regulated chromosomal passenger protein and its nuclear accumulation is linked to poor prognosis for human gastric cancer. Exp Cell Res. 2006;312(7):962-973. doi:10.1016/j. yexcr.2005.12.015

29. Bi N, Sun Y, Lei S, et al. Identification of 40 S ribosomal protein S8 as a novel biomarker for alcohol-associated hepatocellular carcinoma using weighted gene co-expression network analysis. Oncol Rep. 2020;44(2):611-627. doi:10.3892/or.2020.7634

30. Gao X, Wen X, He H, et al. Knockdown of CDCA8 inhibits the proliferation and enhances the apoptosis of bladder cancer cells. PeerJ. 2020;8:e9078. doi:10.7717/peerj.9078

31. Yang WX, Pan YY, You CG. CDK1, CCNB1, CDC20, BUB1, MAD2L1, MCM3, BUB1B, MCM2, and RFC4 may be potential therapeutic targets for hepatocellular carcinoma using integrated bioinformatic analysis. Biomed Res Int. 2019;2019:1245072. doi: $10.1155 / 2019 / 1245072$

32. Kitajima S, Li F, Takahashi C. Tumor milieu controlled by RB tumor suppressor. Int $J$ Mol Sci. 2020;21(7):2450. doi:10.3390/ ijms 21072450

33. Manicum T, Ni F, Ye Y, et al. Prognostic values of E2F mRNA expression in human gastric cancer. Biosci Rep. 2018;38(6). doi:10.1042/BSR20181264

34. Chen HZ, Tsai SY, Leone G. Emerging roles of E2Fs in cancer: an exit from cell cycle control. Nat Rev Cancer. 2009;9(11):785-797. doi:10.1038/nrc2696 
35. Casimiro MC, Crosariol M, Loro E, et al. Cyclins and cell cycle control in cancer and disease. Genes Cancer. 2012;3(1112):649-657. doi:10.1177/1947601913479022

36. Lepère-Douard C, Trotard M, Le Seyec J, et al. The first transmembrane domain of the hepatitis B virus large envelope protein is crucial for infectivity. $J$ Virol. 2009;83(22):11819-11829. doi:10.1128/JVI.01026-09

37. Gao Q, Qiu S-J, Fan J, et al. Intratumoral balance of regulatory and cytotoxic T cells is associated with prognosis of hepatocellular carcinoma after resection. $J$ Clin Oncol. 2007;25(18):2586-2593. doi:10.1200/JCO.2006.09.4565
38. Gentles AJ, Newman AM, Liu CL, et al. The prognostic landscape of genes and infiltrating immune cells across human cancers. Nat Med. 2015;21(8):938-945. doi:10.1038/nm.3909

39. Franklin RA, Liao W, Sarkar A, et al. The cellular and molecular origin of tumor-associated macrophages. Science. 2014;344 (6186):921-925. doi:10.1126/science.1252510

\section{Publish your work in this journal}

The International Journal of General Medicine is an international, peer-reviewed open-access journal that focuses on general and internal medicine, pathogenesis, epidemiology, diagnosis, monitoring and treatment protocols. The journal is characterized by the rapid reporting of reviews, original research and clinical studies across all disease areas. The manuscript management system is completely online and includes a very quick and fair peer-review system, which is all easy to use. Visit http://www.dovepress.com/ testimonials.php to read real quotes from published authors. 